\title{
Role of Mean Platelet Volume As An Adjunct in Evaluation of Acute Inflammation
}

\author{
Nabila Afsar*, Idrees Akhtar, Afroze, Habeebunnisa Tahniath, Zakia Abid
}

Department of Pathology, Deccan College of Medical Sciences, Kanchan Bagh, Hyderabad. India

\section{ABSTRACT}

Background: Platelets are also known for their role in the pathophysiology of vascular inflammation. Activated platelets can promote vascular inflammation, causing endothelial inflammation and subsequent leucocyte extravasation. Increased MPV may be used as a marker of platelet activation. We aim to study the relationship of MPV with total WBC count in cases with neutrophilic leucocytosis and to assess if MPV may have any role as an inflammatory marker.

Methods: A retrospective study was conducted including 97 cases of leucocytosis with neutrophilia. 95 age and sex matched healthy individuals were used as controls. MPV values and total WBC counts were obtained on automated cell counter Horiba pentra ES60 and manual differential count was performed.

Results: MPV did not show any significant change in the study group compared to the control group. MPV was noted to be higher in cases with associated thrombocytopenia, while MPV was lower in cases with thrombocytosis.

Conclusion: MPV has no direct role in the evaluation of acute inflammation. However the increased MPV noted in cases of thrombocytopenia with neutrophilic leucocytosis suggests that activation of platelets causes a rise in MPV due to shape change and may be implicated in impending sepsis and disseminated intravascular coagulation due to the production of thromboxane A2 by activated platelets, warranting the need for further workup and prospective studies in order to establish the role of MPV as a marker for impending sepsis and DIC in cases with neutrophilic leucocytosis.

Keywords: MPV, Mean Platelet Volume, Acute Inflammation, Neutrophilic Leukocytosis, Platelet Activation

\section{Introduction}

Platelets are known for their role in the pathophysiology of vascular inflammation apart from their role in primary hemostasis and arterial thrombus formation. Activated platelets can promote vascular inflammation, causing endothelial inflammation and subsequent leucocyte extravasation via their stored cytokines and chemokines. ${ }^{[1]}$ With the availability of platelet parameters in routine complete blood count reports by automated blood cell counters, these newer parameters may have an additional diagnostic and prognostic significance in various clinical conditions. Mean platelet volume is a potential marker of platelet reactivity and is calculated by dividing the plateletcrit by the number of platelets. MPV may be determined in the progenitor cell, the bone marrow megakaryocyte. It is presumed that young platelets, those released recently from bone marrow are larger and more dense, contain more of certain proteins (i.e. PF4), and exhibit some alterations in function compared to smaller platelets. Circulating platelets may, indeed diminish in size during a normal circulating lifespan by shedding some of their surface components. ${ }^{[2]}$ The platelet volume is found to be associated with cytokines (thrombopoietin, interleukin-6 and interleukin-3) that regulate megakaryocyte ploidy and platelet number and result in the production of larger platelets. ${ }^{[2,3,4]}$ Increased MPV indicates increased platelet diameter, which can be used as a marker of production rate and platelet activation. During activation, platelets' shapes change from biconcave discs to spherical, and a pronounced pseudopod formation occurs that leads to MPV increase. ${ }^{[5]}$

In this study, we aim to study the relationship of mean platelet volume with total white blood cell count in conditions resulting in neutrophilic leucocytosis and to assess if mean platelet volume may have any role as an inflammatory marker.

\section{Materials and Methods}

A retrospective study was conducted with retrieval of data from the data registers and electronic data base of the automated cell counter, during the months of June to July 2016 and included all inpatient and outpatient cases of leucocytosis with neutrophilia (WBC count above $11 \times 10^{3} / \mathrm{cmm}$ and neutrophils more than $75 \%$ ). CBC values were obtained by Horiba Pentra ES60 following the standard operating procedure of sample collection in EDTA vacuotainers, stringent quality control maintenance 
and immediate processing of sample within 2 hours. The differential count was performed manually by counting 100 cells in a Leishman stained slide. 97 cases were included in the study, all showing neutrophilic leucocytosis - Complete blood counts of 95 healthy individuals with normal complete blood counts including 45 males and 50 females of age groups ranging from 18 to 70 years were used as controls.

Inclusion Criteria: $\mathrm{CBC}$ of all cases with neutrophilic leucocytosis with WBC count $>11 \times 10^{3} / \mathrm{cmm}$ and neutrophils more than $75 \%$.

Exclusion criteria: 1) Paediatric and Octogenarian patients; 2) Patients with very high WBC count above $50 \times 10^{3} / \mathrm{cmm}$. 3) Patient with high WBC count without associated neutrophilia. 4) Patients with haematological malignancies and 5) Antenatal patients

CBC reports of the 95 patients with neutrophilic leucocytosis were analysed and compared with the control group. Statistical analysis using mean, standard deviation, range was done using Microsoft excel and student ' $t$ ' test was done to obtain the $\mathrm{p}$ value to compare with the control group and establish the statistical significance.

\section{Results}

Ninety five cases of neutrophilic leucocytosis were analysed out of which 53(55.8\%) were males and 42(52.6\%) were females in the ratio of 1.2:1. 95 controls were also analysed including 45(47.4\%) males and 50(52.6\%) females. (Table 1) The mean age of subjects was 48.8 years and ranged from 16 to 79 years. Mean age of controls is 36.1 years and ranged from 18 to 70 years.

The mean WBC count noted in the cases with neutrophilic leucocytosis was $17.9 \times 10^{3} / \mathrm{cmm}$ and ranged from 11.4 to $38 \times 10^{3} / \mathrm{cmm}$ with a Standard Deviation of 5.77. Platelet count ranged from 21 to $682 \times 10^{3} / \mathrm{cmm}$ with a mean of $271.4 \times 10^{3} / \mathrm{cmm}$ and Standard deviation of 118.4 .

The mean platelet volume in the 95 subjects with neutrophilic leucocytosis ranged from $6.5-11.5 \mu \mathrm{m}^{3}$, with an average of 8.28 and SD of 1.02, while the MPV was 8.23 with SD of 0.8 and ranged from 6.8-10.6. There was no statistically significant difference noted in the mean platelet volume noted in the 2 groups.(Table 2)

The mean platelet volume was assessed in relation with the rising $\mathrm{WBC}$ count and showed no significant increase or decrease in the MPV with increasing WBC count . (Table 3)

The mean platelet volume was assessed in relation with platelet counts and it was found that MPV was significantly higher in thrombocytopenic patients with neutrophilic leucocytosis, compared to those cases with normal platelet reference range $(\mathrm{p}=0.006)$. It was also noted that cases with thrombocytosis had a statistically significantly lower MPV when compared with cases with normal platelet reference range. $(p=0.004)$ (Table 4$)$

Table 1: Patient Demographic Data.

\begin{tabular}{|l|c|c|c|c|c|c|}
\hline \multirow{2}{*}{ AGE } & \multicolumn{3}{|c|}{ CASES } & \multicolumn{3}{c|}{ CONTROLS } \\
\cline { 2 - 6 } & Male & Female & Total & Male & Female & Total \\
\hline $16-25$ & $5(5.3 \%)$ & $9(9.5 \%)$ & $14(14.7 \%)$ & $15(15.7 \%)$ & $22(23.2 \%)$ & $37(38.9 \%)$ \\
\hline $26-35$ & $5(5.3 \%)$ & $8(8.4 \%)$ & $13(13.7 \%)$ & $6(6.3 \%)$ & $9(9.5 \%)$ & $15(15.8 \%)$ \\
\hline $36-45$ & $8(8.4 \%)$ & $2(2.1 \%)$ & $10(10.5 \%$ & $7(7.4 \%)$ & $7(7.4 \%)$ & $14(14.7 \%)$ \\
\hline $46-55$ & $12(12.6 \%)$ & $8(8.4 \%$ & $20(21 \%)$ & $8(8.4 \%)$ & $3(3.2 \%)$ & $11(11.6 \%)$ \\
\hline $56-65$ & $11(11.6 \%)$ & $8(8.4 \%)$ & $19(20 \%)$ & $5(5.3 \%)$ & $8(8.4 \%)$ & $13(13.7 \%)$ \\
\hline$>66$ & $12(12.6 \%)$ & $7(7.5 \%)$ & $19(20 \%)$ & $4(4.2 \%)$ & $1(1.1 \%)$ & $5(5.3 \%)$ \\
\hline Total & $\mathbf{5 3 ( 5 5 . 8 \% )}$ & $\mathbf{4 2 ( 4 4 . 2 \% )}$ & $\mathbf{9 5}(\mathbf{1 0 0} \%)$ & $\mathbf{4 5 ( 4 7 . 4 \% )}$ & $\mathbf{5 0 ( 5 2 . 6 \% )}$ & $\mathbf{9 5}(\mathbf{1 0 0} \%)$ \\
\hline
\end{tabular}

Table 2: Mean platelet volume in cases versus controls.

\begin{tabular}{|l|c|c|c|}
\hline MPV $\left(\mu \mathrm{m}^{3}\right)$ & Cases $(\mathrm{n}=95)$ & Control(n=95) & P value \\
\cline { 1 - 3 } Range & $6.5-11.5$ & $6.8-10.6$ & 8.23 \\
\cline { 1 - 3 } Mean & 8.28 & 0.80 & \multirow{2}{*}{0.74} \\
\hline Standard deviation & 1.02 & 0.80 & \\
\hline
\end{tabular}

Table 3: Mean platelet volume in relation with WBC in cases with neutrophilic leucocytosis.

\begin{tabular}{|l|c|c|c|}
\hline WBC count $\mathbf{x} \mathbf{1}^{3} / \mathbf{c m m}$ & MPV $\left(\mu \mathbf{m}^{3}\right)$ & Range & SD \\
\hline 11 to $15(n=41)$ & 8.1 & $6.5-10.5$ & 0.9 \\
\hline 16 to $20(n=29)$ & 8.3 & $6.7-11.5$ & 1.2 \\
\hline 21 to $25(n=15)$ & 8.6 & $7.3-10.4$ & 1.1 \\
\hline 26 to $30(n=8)$ & 8.6 & $6.8-9.3$ & 0.8 \\
\hline 31 to $40(n=4)$ & 8.4 & $7.2-9.2$ & 0.9 \\
\hline
\end{tabular}


Table 4: Mean platelet volume in relation with platelet count in cases with neutrophilic leucocytosis.

\begin{tabular}{|l|c|c|c|}
\hline Platelet count & MPV $\left(\mu \mathrm{m}^{3}\right)$ & Range & SD \\
\hline $21-149(n=12)$ & 9.56 & $8.2-11.5$ & 1.0 \\
\hline $150-459(n=76)$ & 8.16 & $6.5-10.5$ & 0.87 \\
\hline$>460(n=7)$ & 7.3 & $6.7-8.1$ & 0.47 \\
\hline
\end{tabular}

\section{Discussion}

The study was aimed to demonstrate if mean platelet volume could be used as an adjunct to evaluate patients with acute inflammation considering the fact that platelets play an important role in the pathophysiology of vascular inflammation. PLTs have not only hemostatic functions but also are considered as inflammatory anucleate cells because many studies demonstrated that PLT counts and PLT parameters are strongly associated with inflammatory and infectious condition. Platelet parameters can be easily obtained on a routine cell analyser report and could be used as a potential marker of platelet reactivity and activation. There is a well-known close relationship between leukocytes and platelets especially in inflamed endothelium . Leukocytes can roll on a template of adherent platelets, firmly adhere, and then transmigrate through the adherent platelets. ${ }^{[6]}$ It is thought that PLTs are one of the first responding anucleate cells during the development of sepsis. Platelet activation readouts have been suggested as biomarkers for the development of septic complications and have been related to prognosis. ${ }^{[7]}$

In the present study, we found that MPV did not show any statistically significant change with increasing WBC count, these findings were concordant with the study by Ozturk $\mathrm{N}$ et al who studied the changes in platelet parameters in leucocytosis and found that the MPV and PDW did not significantly differ in these patients. ${ }^{[8]}$ Gurler $\mathrm{M}$ et al suggested that either increase or decrease in MPV in inflammatory conditions should be the result of the effects of inflammatory cytokines in bone marrow. ${ }^{[9]}$ Activated platelets become larger in these conditions and cause an elevation in MPV values in hemogram tests. However, at an uncertain level, MPV tend to be decreased after utilization of these activated larger platelets in inflammatory processes thus, remaining smaller platelets lead a decrease in MPV in blood count tests.

Z.A. Özturk et al found that in patients with active UC and $\mathrm{CD}$, there was a statistically significant decrease in MPV, PDW levels and increase in PCT levels when compared to healthy controls. In remission phase of IBD while MPV levels were lower, PDW and PCT levels were higher than control group. There was statistically significant change in all platelet indices during diseases follow-up. ${ }^{[10]}$

Zhe Fan et al showed that MPV was clearly lower in patients with acute gangrenous appendicitis. However, the MPV did not have a higher sensitivity compared with PDW. ${ }^{[11]}$
Koç et al demonstrated that MPV level was significantly higher in Chronic recurrent sinusitis compared to control group and PLT level was borderline higher in CRS group compared to control group. ${ }^{[12]}$

S.Bozkur et al showed that there was no statistically significant difference between patients with appendicitis (complicated or uncomplicated) and those having no appendicitis with respect to MPV level. However, the complicated appendicitis group had a lower MPV value compared to other groups. ${ }^{[13]}$ Zareifar et al studied the change in platelet count and mean platelet volume during infectious and inflammatory diseases and demonstrated a higher level of platelet count and lower MPV in the patients with active disease compared to the recovered patients. These parameters were well correlated with the known disease activity markers. ${ }^{[14]}$

An increase in MPV, a sign of larger PLT size, usually is indicative of compensated bone marrow PLT production following stress-induced platelet destruction, as septic shock develops, in fact, the MPV is inversely proportional to the degree of PLT maturity. ${ }^{[15]}$ The present study demonstrated that MPV was significantly higher in thrombocytopenic patients with neutrophilic leucocytosis, compared to those cases with normal platelet reference range $(p=0.006)$. This may be suggestive of impending sepsis. According to McMillan R, morphologically large platelets are seen on blood smears in patients with severe thrombocytopenia, thus suggesting that "hyperfunctional" platelets compensate for low numbers by their increased effectiveness. ${ }^{[16.17]}$ This could explain the increase in MPV noted in our study.

It was also noted in the present study that cases with thrombocytosis had a statistically significantly lower MPV when compared with cases with normal platelet reference range $(p=0.004)$. Van der Lelie et al and Robbins et al also observed that patients with reactive thrombocytosis had considerably lower mean platelet volumes than those with normal subjects, which was similar to the observation in the present study. ${ }^{[18,19]}$

While platelets can have a beneficial role in host response to an invading pathogen, during sepsis, platelet activation contributes to the development of complications such as DIC, multiple organ failure, acute lung injury (ALI) and acute kidney injury (AKI). ${ }^{[20]}$ This observation highlights 
the need for a marker of platelet activation to facilitate early prevention and management of complications in patients with acute inflammation with impending sepsis.

\section{Conclusion}

In patients with neutrophilic leucocytosis suggesting acute inflammation due to varying etiology, MPV did not show any significant change compared to the control group of healthy individuals with normal WBC count. However, MPV was noted to be higher in those cases which had associated thrombocytopenia, while the MPV was lower in cases with thrombocytosis. The study infers that although MPV has no direct role in the evaluation of acute inflammation, the increased MPV noted in cases of thrombocytopenia with neutrophilic leucocytosis suggests that activation of platelets causes a rise in MPV due to shape change and may be implicated in impending sepsis and disseminated intravascular coagulation due to the production of thromboxane A2( a potent inducer of platelet activation) by activated platelets. This finding warrants the need for further workup and studies in order to establish the role of mean platelet volume as a marker for impending sepsis and DIC in cases with neutrophilic leucocytosis.

\section{Acknowledgment}

We would like to thank our technical staff who have of utmost help in the collection of data

\section{References}

1. 1. Projahn D, Koenen RR. Platelets. Key players in vascular inflammation. J Leukoc Biol. 2012;92(6):1167-1175.

2. 2. Larsen SB, Grove EL, Hvas AM, Kristensen SD. Platelet turnover in stable coronary artery disease-influence of thrombopoietin and low-grade inflammation. PLoS One 2014;9:e85566.

3. Brown AS, Hong Y, de Belder A, Beacon H, Beeso J, Sherwood R, et al. Megakaryocyte ploidy and platelet changes in human diabetes and atherosclerosis. Arterioscler Thromb Vasc Biol 1997; 17:802-7.

4. Osselaer JC, Jamart J, Scheiff JM. Platelet distribution width for differential diagnosis of thrombocytosis. Clin Chem 1997;43:1072-76.

5. Budak YU, Polat M, Huysal K. The use of platelet indices, plateletcrit, mean platelet volume and platelet distribution width in emergency non-traumatic abdominal surgery: a systematic review. Biochemia Medica. 2016;26(2):178-193.
6. Smyth SS, McEver RP, Weyrich AS et al. 2009 Platelet Colloquium Participants: Platelet functions beyond hemostasis. J Thromb Haemost. 2009; 7(11)

7. Gao Y, Li Y, Yu X et al. The impact of various platelet indices as prognostic markers of septic shock. PLoS One. 2014; 9(8)

8. Ozturk N, Baygutalp NK, Bakan E, Altas GF, Polat H, Dorman E. Changes in platelet parameters in leukocytosis. The Pan African Medical Journal. 2016;24:185.

9. Gurler, Mujgan; AKTAS, Gulali. A review of the association of mean platelet volume and red cell distribution width in inflammation. Int J Res Med Sci, 2016 4(1) 1-4.

10. Özturk ZA, Dag MS, Kuyumcu ME, et al. Could platelet indices be new biomarkers for inflammatory bowel diseases? Eur Rev Med Pharmacol Sci; 2013;17:334-341

11. Fan Z, Pan J, Zhang Y, et al., "Mean Platelet Volume and Platelet Distribution Width as Markers in the Diagnosis of Acute Gangrenous Appendicitis," Disease Markers, 2015,(2015),1-4.

12. Koç S, Eyibilen A, Erdoğan AS. Mean Platelet Volume as an Inflammatory Marker in Chronic Sinusitis, Eur J Gen Med 2011;8(4):314-317

13. Bozkurt S, Köse A, Erdogan, et al. MPV and other inflammatory markers in diagnosing acute appendicitis. JPakMedAssoc; 2015;65(6): 637-641

14. Zareifar S., Farahmand Far MR, Golfeshan F, Cohan, N. Changes in Platelet Count and Mean Platelet Volume During Infectious and Inflammatory Disease and Their Correlation With ESR and CRP. J. Clin. Lab. Anal., 2014;28: 245-248.

15. Van der Lelie J, Von dem Borne AK. Increased mean platelet volume in septicaemia. J Clin Pathol 1983;36: 693-696

16. McMillan R. Therapy for adults with refractory chronic immune thrombocytopenic purpura.Ann Intern Med 1997; 126:307-314.

17. Kaushansky K , Gerald J Roth. Megakaryocytes and platelets. In: John P Greer, John Foerster, John N Lukens, George M Rodgers, Frixos Paraskevas, Bertil Glader, editors. Wintrobe's clinical Hematology. 11th edition. Philadelphia. Lippincott Williams \& Wilkins; 2004: 632

18. Van der Lelie J, Von dem Borne AK. Platelet volume analysis for differential diagnosis of thrombocytosis. Journal of Clinical Pathology. 1986;39(2):129-133.

19. Robbins G, Barnard DL. Thrombocytosis and microthrombocytosis: a clinical evaluation of 372 cases. Acta Haematol 1983;70:175-82.

20. de Stoppelaar SF, van 't Veer C, van der Poll T. The role of platelets in sepsis. Thrombosis and Haemostasis 2014; 112: 627-842.

*Corresponding author:

Dr. Nabila Afsar, Department of Pathology, Deccan college of Medical Sciences, DMRL X Road, Santoshnagar, Kanchan Bagh,

Hyderabad, Telengana- 500058, India.

Phone: +91 9848020386

Email: nabila_dr@yahoo.com

Date of Submission : 19.04.2017

Date of Acceptance : 23.05.2017

Financial or other Competing Interests: None.

Date of Publication : 31.08.2017 